\title{
Modelando la Varianza de la Forma: Morfometría Geométrica Aplicaciones en Biología Evolutiva
}

\author{
Modelling Shape Variance: Geometric Morphometric Applications in Evolutionary Biology
}

\author{
Hugo A. Benítez ${ }^{*, * *}$ \& Thomas A. Püschel ${ }^{* * *}$
}

BENÍTEZ, H. A. \& PÜSCHEL, T. A. Modelando la varianza de la forma: morfometría geométrica aplicaciones en biología evolutiva. Int. J. Morphol., 32(3):998-1008, 2014.

RESUMEN: La comparación de caracteres anatómicos entre organismos ha sido un elemento central de la biología comparada. Históricamente, la clasificación taxonómica y la comprensión de la diversidad biológica se han basado en descripciones morfológicas. En base a una revolución matemática cuantitativa, el estudio de la morfología ha tenido un importante énfasis gracias al desarrollo del análisis de la forma mediante la combinación de métodos estadísticos multivariados y nuevas maneras de visualización. El objetivo de la presente revisión es dar una visión actualizada sobre los avances del estudio de la morfometría geométrica (MG) en biología evolutiva, así como introducir a temáticas en fuerte desarrollo (e.g. estabilidad del desarrollo, integración y modularidad morfológicas, entre otras). Se espera proporcionar una visión amplia del uso de la MG en biología evolutiva, destacando la necesidad de aumentar el esfuerzo de investigación en esta disciplina, junto con llamar la atención acerca de la utilidad de la MG como una herramienta efectiva, precisa, amigable y barata para cuantificar y estudiar la variación morfológica.

PALABRAS CLAVE: Morfometría geométrica; Biología evolutiva; Integración; Modularidad; Asimetría fluctuante; Dimorfismo sexual.

\section{INTRODUCCIÓN}

La comparación de caracteres anatómicos entre organismos ha sido un elemento central de la biología comparada durante siglos. Históricamente, la clasificación taxonómica y la comprensión de la diversidad biológica han basado sus fundamentes estructuralmente en descripciones morfológicas (Adams et al., 2004). A principios del siglo $\mathrm{XX}$, la biología comparada entró en una transición entre el campo descriptivo y la ciencia cuantitativa, en la que el análisis morfológico tuvo una similar revolución cuantitativa (Bookstein, 1998).

Sobre la base de esta revolución matemática cuantitativa, el estudio de la morfología ha tenido un importante énfasis gracias al desarrollo estadístico del análisis de la "forma"; esto hizo posible la combinación de métodos estadísticos multivariados y nuevas maneras de visualizar una estructura (Adams \& Funk, 1997). Esta "Síntesis Morfométrica", conocida actualmente como "Morfometría Geométrica" (MG), permite un máximo aprovechamiento de la información geométrica que posee una estructura (Rohlf
\& Marcus, 1993). Estas herramientas permiten el estudio de la forma integrando el tamaño de los organismos, proporcionando análisis robustos y herramientas gráficas para la cuantificación y visualización de la variación morfológica intra e interespecífica (Adams et al., 2013).

La MG permite el estudio de la forma, definida como las propiedades geométricas restantes tras remover los efectos de la escala, la rotación y la traslación de un objeto (Fig. 1) (Adams \& Funk; Rohlf et al., 1996; Rohlf \& Slice, 1990). Una técnica dentro de estos métodos de evaluación de la forma, es la búsqueda de componentes no-uniforme del cambio de la forma (Thin Plate Spline), la cual representaría todos los movimientos de los hitos, es decir, las variaciones locales y no lineales, indicando por tanto los cambios producidos en sectores puntuales de la forma. Por lo tanto, la MG ofrecería una mejor interpretación biológica y constituiría una herramienta gráfica para la visualización y cuantificación de la variación morfológica en diferentes contextos ecológicos y evolutivos (Adams \& Rohlf, 2000;

\footnotetext{
* Faculty of Life Sciences, University of Manchester, Michael Smith Building, Oxford Road, Manchester M13 9PT, UK

** Instituto de Alta Investigación, Universidad de Tarapacá, Casilla 7-D Arica CHILE

**** Centre for Anatomical and Human Sciences, Hull York Medical School, University of York, Heslington, York YO10 5DD, UK
} 


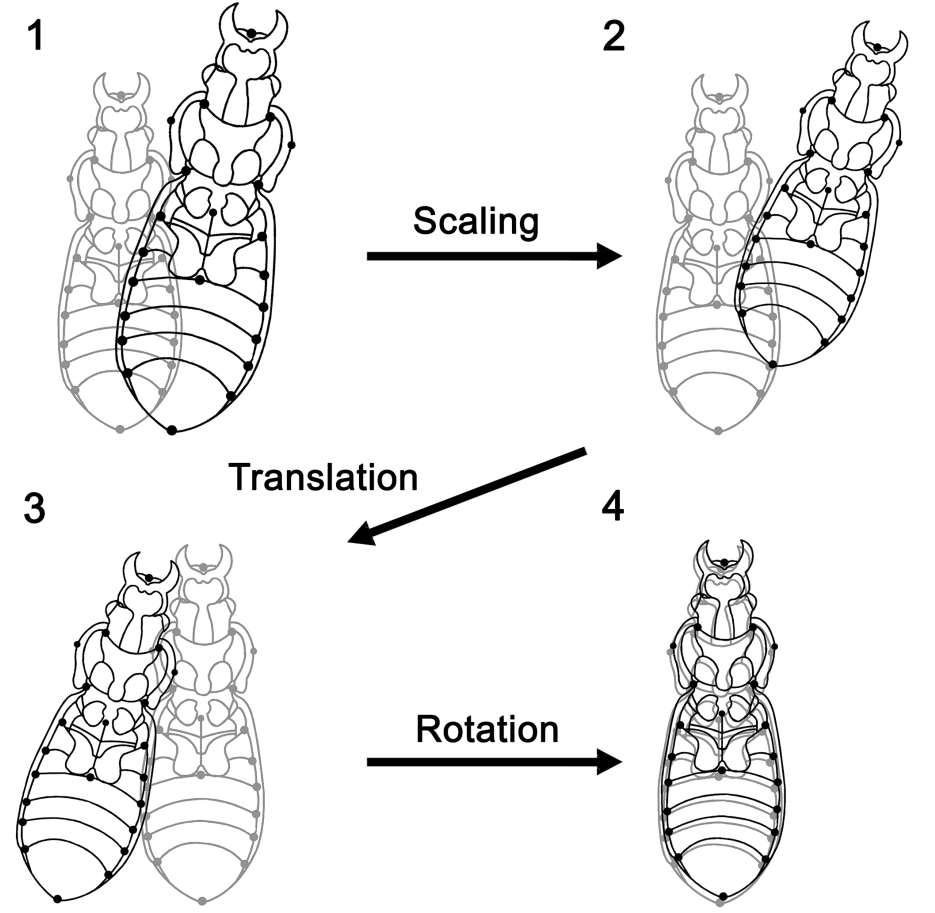

Fig. 1. Pasos de la superposición de Procusto ejemplificado en el cuerpo ejemplo en Ceroglossus: 1) escalamiento a un mismo tamaño de centroide, 2) traslación a un centroide común, y 3) rotación para minimizar la suma de distancias cuadradas entre los hitos correspondientes.

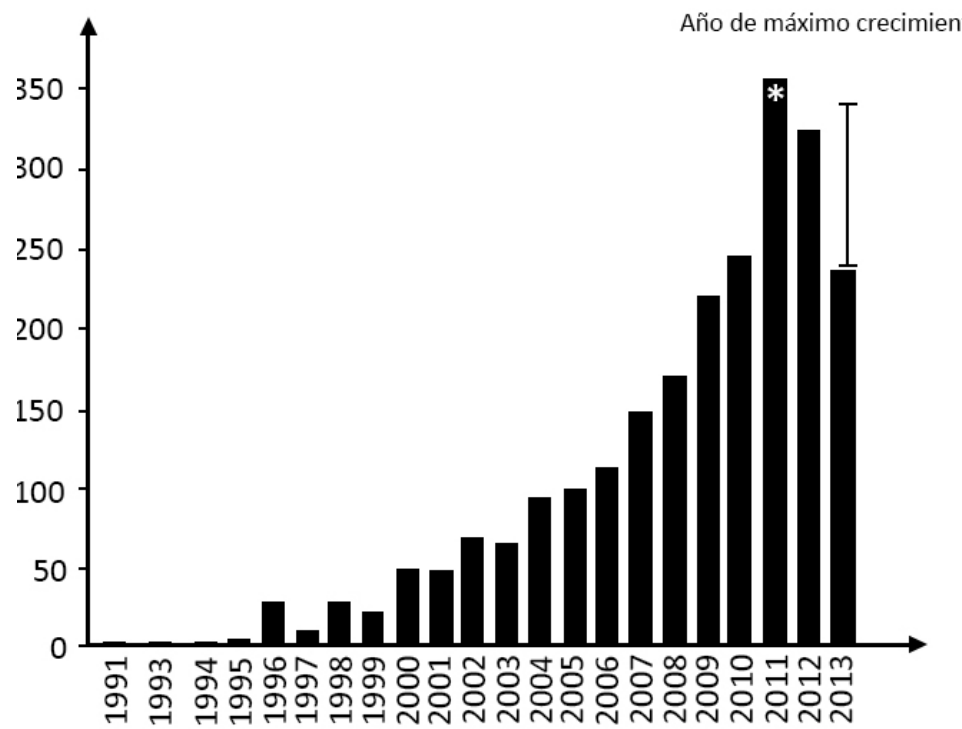

Fig. 2. Número de artículos en morfometría geométrica publicados al año 2013 (registros de Web of Science).

Alibert et al., 2001; Benítez De La Fuente et al., 2010). Estos avances en el estudio de la morfología, contribuyeron en un aumento significativo del conocimiento en estudios taxonómicos, ecológicos, de la definición de especie frente a la dispersión geográfica y en estudios de diferencia- ción sexual (Alibert et al.; Bertin et al., 2002). Sin embargo, pese a la potencialidad de estas herramientas, los estudios de variación morfológica han recibido una atención moderada (Brown et al., 1992; Thomas et al., 1998; Adams et al., 2004), principalmente por la carencia de exactitud estadística en los métodos de morfometría tradicional a nivel intraespecífico. A pesar de esta carencia, varios autores han proporcionado revisiones del uso de la MG en diferentes áreas de la ciencia (Rohlf \& Slice; Bookstein, 1991; Rohlf \& Marcus; O'Higgins, 2000; MacLeod \& Forey, 2002; Adams et al., 2004; Slice, 2007; ToroIbacache et al., 2010; López et al., 2012; ParésCasanova \& Martínez, 2013; Rios-Rodas et al., 2013; Klingenberg, 2013; Adams et al., 2013).

De hecho existe una creciente utilización de estos métodos, siendo evidenciable en los registros de ISI Web of Knowledge, que a la fecha señalan 5582 artículos publicados con la presencia de la palabra "geometric morphometrics", existiendo así un aumento exponencial en los últimos 5 años que llega a más de 300 (353 año 2011) artículos anuales (Fig. 2).

Orígenes y Definiciones . Los orígenes de la MG nacen motivados en parte, por los estudios en las grillas deformadas de Thompson (1942). El desarrollo de nuevas propiedades basadas en una teoría matemática coherente, capaz de capturar la forma, hace que esta nueva morfometría haya sido denominada como geométrica. Su recepción fue acogida como una "revolución" para el mundo del análisis morfológico, debido a la gran potencialidad y poder analítico de este nuevo método (Rohlf \& Marcus).

Los cimientos de la MG se basan en una matemática relativamente compleja (basada enteramente en el álgebra matricial), y aunque sus objetivos y su manera de proceder son intuitivos (es una herramienta diseñada para proveer resultados visuales), es necesario esclarecer sus fundamentos, aunque por limitaciones de espacio será de manera sucinta. En la última década han sucedido grandes e importantes avances en el desarrollo de esta materia, los cuales han permitido generalizar ciertos estándares teórico-prácticos. Trabajos como 
los de Bookstein (1991) sobre la base histórica y el desarrollo de las técnicas, Goodall (1991) en particular para la metodología Procusto (Dryden \& Mardia, 1998) quienes realizan una síntesis detallada de la estadística aplicable al estudio de la forma, Small (1996) quien da un estudio detallado de la noción y tipos de espacios de forma, son algunos de los ya clásicos desarrollos en MG. Posteriormente, Klingenberg \& McIntyre (1998) desarrollaron métodos para el estudio de patrones de asimetría mediante MG, junto con otros múltiples autores que han contribuido en la estructuración de la tecnica (McLeod \& Forey; Polak, 2003; Hallgrímsson \& Hall, 2005; Zelditch et al., 2012). Para una introducción detallada al campo de la MG, ver Zelditch et al. (2012).

\section{Morfometría Tradicional vs. Morfometría Geométrica.} La morfometría lineal se caracteriza por la utilización de la estadística multivariada a problemas relativos a la forma de los organismos (Marcus, 1990). No obstante a diferencia de la MG, sus datos primarios no son coordenadas cartesianas, sino que distancias, índices o ángulos (Tornese \& Nabar, 2013; Cantin et al., 2013). Estos métodos rara vez preservan las relaciones geométricas del objeto de estudio durante el análisis, lo que dificulta enormemente la visualización de los cambios morfológicos. Como consecuencia de lo anterior, la generación de gráficos o visualizaciones de los resultados usualmente se hace a través de tablas poco intuitivas. Otra importante diferencia surge en relación a la estandarización de las variables. Aunque las distancias son inherentemente independientes de la posición y orientación del objeto del cual son obtenidas, no permiten ninguna remoción de los efectos de la escala/tamaño matemáticamente comparable a la obtenida en MG mediante el análisis de Procusto.

En general las estandarizaciones utilizadas en morfometría lineal, van desde la utilización de índices y proporciones, a la transformación de las variables de distintas maneras. El problema de los índices reside en la carencia de una estadística bien desarrollada para su tratamiento multivariado, así como una artificiosa disminución de la varianza (Atchley et al., 1976). Las distancias por su parte poseen la limitación de que resulta complicado preservar la información relacionada a las posiciones relativas de los hitos entre sí. Por ejemplo podrían existir múltiples formas con un mismo valor de distancia entre sus puntos más distales, sin que estas distancias sea equivalentes u homologas. Para solucionar problemas como el anterior, muchas veces se ha propuesto al análisis multivariado conjunto de distancias y valores angulares entre tres hitos (estos últimos poseen la cualidad de ser invariantes a la localización, orientación e incluso el tamaño) (Slice, 2005). Combinando los valores de las distancias utilizadas para construir un ángulo, junto con su respectivo valor angular, permite preservar las posi- ciones relativas de los hitos entre sí. No obstante, mezclar datos medidos en diferentes niveles de medición, puede ocasionar problemas en algunos análisis multivariados, como aquellos basados en matrices de varianza-covarianza. En estos casos se recomienda la utilización de la matriz de correlación, pues permite una estandarización de los niveles de medición, pese a que continúa haciendo el análisis y la visualización, aún más abstractos. Otra solución propuesta es calcular todas las distancias inter-hitos existentes (idea sobre la que se fundamenta el enfoque morfométrico (Euclidean Distances Matrix o EDMA), lo cual también estandarizaría la posición relativa de los hitos, no obstante inflaría el número de variables y grados de libertad (Lele \& Richtsmeier, 2001).

Por su parte para estandarizar la variación debida a tamaño, se ha propuesto interpretar el primer componente (PC1) de un análisis de componentes principales (PCA) como la variable que resume la variación debida al tamaño, mientras que los componentes restantes representarían la variación debida a la forma (MacLeod, 2005). Lo anterior se fundaría una concepción simplista de que el PC1 sería un eje de "solo-tamaño", lo cual es equivocado. El PC1 puede representar variación debida al tamaño, pero este cambio no tiene por qué ser isométrico (Shea, 1985). Únicamente si el primer eje longitudinal posee una pendiente de uno con respecto a todos los demás ejes, se podría considerar que es isométrico-multivariado y que no habría cambio de forma vinculado a cambio de tamaño. Sin embargo, en la mayoría de los casos biológicos (quizás todos), la pendiente es diferente a uno con respecto a los demás ejes, por lo que cualquier cambio en el eje de "tamaño" (PC1), iría acompañado de cambios en la forma. Es por esto, que algunos autores han propuesto que el primer eje sea denominado "eje de forma tamaño-determinado", y no eje de "solo-tamaño" (McKinney \& McNamara, 1991). Otras soluciones propuestas para remover el efecto del tamaño han sido la utilización de puntajes $\mathrm{Z}$ y de variables de la forma de Mosimann (Darroch \& Mosimann, 1985), que consisten en dividir cada variable por la media geométrica de la muestra. Esta estandarización en teoría permitiría remover, al menos parcialmente el efecto del tamaño, aunque sus fundamentos estadísticos no son muy robustos (Darroch \& Mosimann).

Las herramientas de la MG poseen grandes ventajas comparativas en relación a la morfometría lineal, pues entregan una descripción precisa y certera de las formas biológicas, además de permitir una adecuada visualización, interpretación y comunicación de los resultados (Zelditch et al., 2004, 2012). La MG permite visualizar complejas diferencias entre distintas formas biológicas, junto con la puesta a prueba distintas hipótesisestadísticas sustentadas firmemente en una base matemática adecuada. 
Medida de Estabilidad del desarrollo: Asimetría Fluctuante. La estabilidad del desarrollo (ED) se define como la capacidad de un organismo para producir un fenotipo predeterminado por un diseño corporal adaptativo, bajo un conjunto de condiciones genéticas y ambientales específicas (Waddington, 1942). Por tanto, refiere a las capacidades intrínsecas de un individuo para resistir accidentes y perturbaciones durante su crecimiento y desarrollo (Clarke, 1998). La capacidad de un organismo para producir un fenotipo ideal, pese a las perturbaciones encontradas durante el desarrollo, reflejan el mecanismo causal de la ED. Esta capacidad es utilizada para evaluar variedad de tipos de estrés y la capacidad genotípica de corregirlos (Auffray et al., 1999). A menudo se denomina inestabilidad del desarrollo (ID) o ruido del desarrollo (RD) a la tendencia de un sistema del desarrollo para producir cambios morfológicos como respuestas a perturbaciones aleatorias (Klingenberg, 2003; Nijhout \& Davidowitz, 2003).

La herramienta de medición más utilizada para estimar la ED es la Asimetría Fluctuante (AF) (van Valen, 1962; Palmer \& Strobeck, 1986; Clarke; Pither \& Taylor, 2000). La AF es una medida de las pequeñas desviaciones al azar que ocurren entre el lado izquierdo y derecho de rasgos bilateralmente simétricos (van Valen). La principal idea en el uso de la AF como medida de ID, es que el lado izquierdo y derecho (I - D) de un organismo puedan ser vistas como replicas independientes de un mismo desarrollo, donde los lados corporales de un individuo compartirían un mismo genotipo en un ambiente homogéneo (Klingenberg, 2003). En otras palabras, los factores determinantes del desarrollo del lado I y D serian idénticos, por lo que en condiciones ideales se esperaría para éstos una simetría perfecta (Auffray et al.; Klingenberg, 2003).

En los últimos años, la búsqueda de nuevos métodos más precisos para cuantificar la variación de la asimetría en los individuos, ha llevado a la incorporación de la MG como una herramienta que permite visualizar y cuantificar cambios directamente desde la forma, reduciendo la tasa de error de medición presente frecuentemente en las mediciones con morfometría tradicional y permitiendo la estimación de variaciones más sutiles y exactas para el cálculo de asimetría (Klingenberg \& McIntyre; Auffray et al.; Zelditch et al., 2008; Debat et al., 2009; Benítez, 2013, Bravi \& Benítez, 2013). White \& Searle (2008) investigaron el uso de la MG a través de la asimetría fluctuante como proxy morfológico para cuantificar la diversidad genética de musarañas (Sorex araneus) en diferentes islas. Su estudio cuantificó la asimetría del lado izquierdo y derecho de la mandíbula de esta musaraña, en la que encontraron una pequeña relación entre la diversidad genética y la asimetría fluctuante de los individuos. La utilización de esta herramienta ha permitido cuan- tificar otros tipos de variaciones en el desarrollo, tales como cambios en la morfología en relación con la mutación en genes responsables del desarrollo. Fondon \& Garner (2007), hipotetizan que en cráneos de perros las simples asimetrías morfológicas son producidas por el incremento de mutaciones en el desarrollo (variaciones en factor de transcripción "Runx2" correlacionado con la sección facial del perro), lo que es confirmado a través de modelos de hitos 3D.

Integración morfológica y modularidad. La integración morfológica, es un fenómeno que se reconoce como la variación coordinada entre rasgos funcionales y del desarrollo de los organismos (Olson \& Miller, 1958), mientras que como contraparte, la modularidad se define como la división de sistemas del desarrollo en componentes parcialmente disociados que se encuentran integrados partir de distintos niveles de organización biológicas (Klingenberg, 2008). El estudio de estas relaciones morfológicas ha tomado alta importancia en los últimos 10 años, debido al creciente interés en el estudio de fenotipos complejos, evo-devo, mapeo genotipo-fenotipo, entre otras temáticas (Pigliucci \& Preston, 2004; Klingenberg, 2003, 2004, 2008). La integración morfológica se estudia mayoritariamente a partir de la estimacion de la covariación de rasgos, pudiendo existir diferentes grados en la fuerza de covariación entre caracteres. Esto seria indicativo de que tan vinculados se encuentran el uno con el otro, por tanto los patrones de covariación se enfocarían en como cambio específicos en los caracteres ocurren coordinadamente. De esta manera la fuerza de integración entre caracteres en morfometría geométrica, se basaría en la distribución o variación de estos cambios sobre las dimensiones en el espacio fenotípico (Klingenberg, 2008). Estudios morfológicos tradicionales de integración se sustentaron en la función de los eigenvalues sobre la matrices de correlación de distancias lineales (Cheverud $e t$ al., 1983; Wagner, 1984, 1990). No obstante, actualmente la mayor parte de estos análisis son desarrollados mediante las herramientas de la morfometría geométrica (Willmore et al., 2006; Young, 2006; Miteroecker \& Bookstein, 2007; Klingenberg, 2008; Goswami \& Polly, 2010; Klingenberg, 2013).

Por otra parte, la modularidad ha sido considerada como un importante principio organizacional de los sistemas biológicos, el cual también se manifestaría a un nivel morfológico (Klingenberg, 2009). Un módulo ha sido tradicionalmente definido como una unidad dentro de la cual existe una alta integración de muchas y/o fuertes interacciones, pero que a su vez son relativamente independientes de otras de esas unidades (Klingenberg, 2008). Dependiendo del problema de investigación, así como del nivel de organización bajo análisis, la naturaleza de las interacciones puede variar (i.e. pueden ser genéticas, del desarrollo y/o funcionales). Desde 
un punto de vista morfométrico, estas interacciones se manifestarían como una fuerte covariación de los componentes dentro de un módulo, y una débil covariación entre distintos módulos (Klingenberg, 2009). Variados estudios en modularidad morfológica han sido desarrollados en los últimos años tanto en aplicación (Klingenberg, 2013), como en el desarrollo teórico de la determinación modular (Goswami \& Polly; Klingenberg, 2013), así como también en la generación de software para el análisis de covariación entre caracteres (e.g coeficiente RV en Klingenberg, 2009)

Por ejemplo, Klingenberg \& Zaklan (2000) utilizan las herramientas de la MG, para evaluar la integración del ala en Drosophila melanogaster, hipotetizando que las alas anteriores y posteriores son unidades del desarrollo que varían de forma independiente. Sus resultados muestran que estos caracteres no varían localmente, concluyendo así que la variación inter-individual, así como las perturbaciones en el desarrollo (AF) modelarían la morfología mediante procesos una integración de los procesos del desarrollo. Por su parte, Klingenberg \& Leamy (2001), utiliza las herramientas de la MG aplicadas a la asimetría fluctuante, con la finalidad de evaluar los efectos en la estabilidad del desarrollo alar en Bombus empatiens. El patrón de covariación de la forma con respecto a la asimetría fluctuante, logró demostrar que los meca-
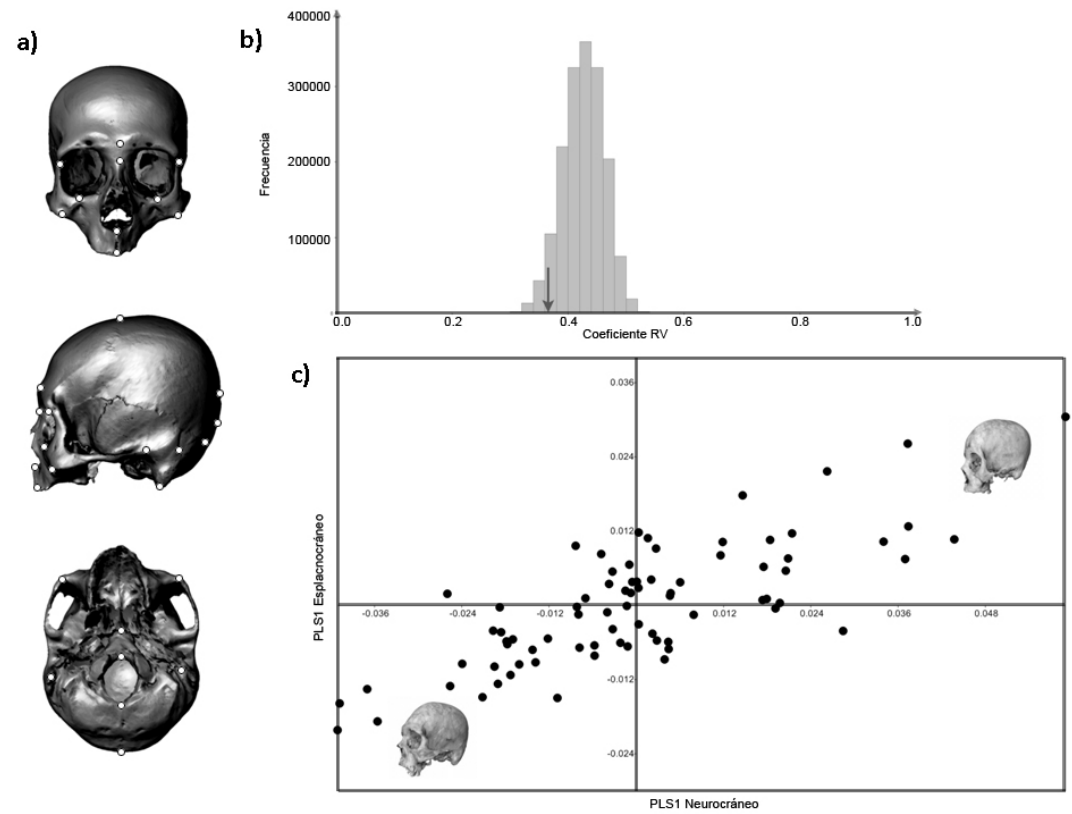

Fig. 3. Ejemplo de aplicación de la MG para el análisis de modularidad e integración morfológicas: a) Hitos 3D in situ sobre un modelo 3D, Arriba: Vista anterior del cráneo mostrando los hitos seleccionados, Centro: Vista lateral de los hitos utilizados, Abajo: Vista inferior de los hitos usados. Los 10 hitos visibles en la vista anterior se usaron para determinar el modulo esplacnocráneal, mientras que los 14 restante definieron al neurocráneo; b) Histograma de la distribución de los coeficientes RV generados por permutación, la flecha muestra que nuestra hipótesis se encuentra en el extremo izquierdo de la curva de distribución, siendo una las partición con el coeficiente RV más bajo (RV: 0,366; porcentaje de particiones alternativas con un valor RV menor al de los módulos hipotéticos: 4,7\%; p-valor tras 1000 rondas de permutación: <0.001); c) Gráfico de covariación del primer par de ejes singulares (PLS1) para los 77 individuos analizados, se observa una integración morfológica moderada entre cara y bóvedas óseas (correlación entre los puntajes del PLS1: 0,78; \% de la covariaza total explicada: 39,4\%; p-valor tras 1000 rondas de permutación: 0,002). nismos del desarrollo morfológico alar se relacionan con el intercambio gaseoso. Otro modelo morfológico comúnmente utilizados en estudios de integración y modularidad han sido las mandíbulas de mamíferos, tales como roedores o murciélagos. Estas se han utilizado tanto para evaluar radiación adaptativa por preferencias nutricionales (Monteiro \& Nogueira, 2010, 2011; Nogueira et al., 2009), como también para medir la independencia relativa de las partes de las mandíbulas en ratones y últimamente también en aves (Klingenbert et al., 2003, Klingenberg \& Marugán-Lobón, 2013).

A modo de ejemplo, en el presente trabajo se analizaron 77 cráneos humanos lo cuales fueron digitalizados utilizando un escáner láser Nextengine Inc., tras lo cual se generaron una serie de modelos tridimensionales en donde 24 hitos tridimensionales fueron colectados (Fig 3.). Se deseó poner a prueba la hipótesis de modularidad del cráneo en base a orígenes embrionarios diferenciales (Ackermann, 2005; Bastir \& Rosas, 2005; Mitteroecker \& Bookstein, 2008). Se estimó el coeficiente RV (análogo multivariado de una correlación) para analizar la independencia relativa de los hipotéticos módulos, y luego se procedió a evaluar el nivel de integración morfológica de estos mismos, mediante un análisis de dos bloques de mínimos cuadrados (PLS) (Klingenberg, 2009). Nuestros resultados confirman el comportamiento modular del neurocráneo y esplacnocráneo (RV: 0.366; $\%$ de particiones alternativas con un valor RV menor al de los módulos hipotéticos: 4,7\%; p-valor tras 1000 rondas de permutación: $<0,001$ ), así como una integración morfológica moderada entre cara y bóvedas óseas (correlación entre los puntajes del PLS1: 0,78; \% de la covarianza total explicada: $39,4 \%$; p-valor tras 1000 rondas de permutación: $0,002)$. Esto puede ser interpretado como que el desarrollo entre neurocráneo y esplacnocráneo es coordinado, no obstante esta coordinación se estructura modularmente, permitiendo una relativa independencia entre las partes. 
Dimorfismo Sexual y Evolución de la Forma. El dimorfismo sexual es una de las fuentes más notables y generalizadas de la variación fenotípica en animales y plantas, por lo cual, a despertado un alto interés en la biología evolutiva (Fairbairn \& Preziosi, 1996). Las diferencias sexuales apreciables en los caracteres morfológicos de machos y hembras son un fenómeno común en muchos taxa de animales, siendo más conspicua la variación en el tamaño y la forma del cuerpo (Benítez De La Fuente et al.; Gannon \& Racz; Galdames \& Zavando, 2012). La historia evolutiva, las vías del desarrollo y el diseño estructural son las principales restricciones de la diversificación fenotípica (Cheverud et al., 1985). Sin embargo, estos tres agentes constituyen un todo integrado debido a que las restricciones histórico-evolutivas y de diseño son epifenómenos de las vías del desarrollo de los organismos (Rieppel, 2006). Estudios de la forma en insectos coleópteros han señalado que el dimorfismo sexual se concentra generalmente en dos secciones de la forma corporal: tanto en la sección abdominal, donde estas variaciones de dimorfismo se asocian son de carácter adaptativo debido a la presencia de huevos en hembras, como en la sección pronotal asociados a la competencia macho-macho producto de variaciones en la proporción sexual en las poblaciones (Benítez De La Fuente et al.; Benítez et al., 2013). Otros estudios han utilizado la variación geométrica de la forma alar en insectos como carácter de dimorfismo, siendo la variación geométrica integrada de las venas se diferencian entre machos y hembras (Benítez et al., 2011).
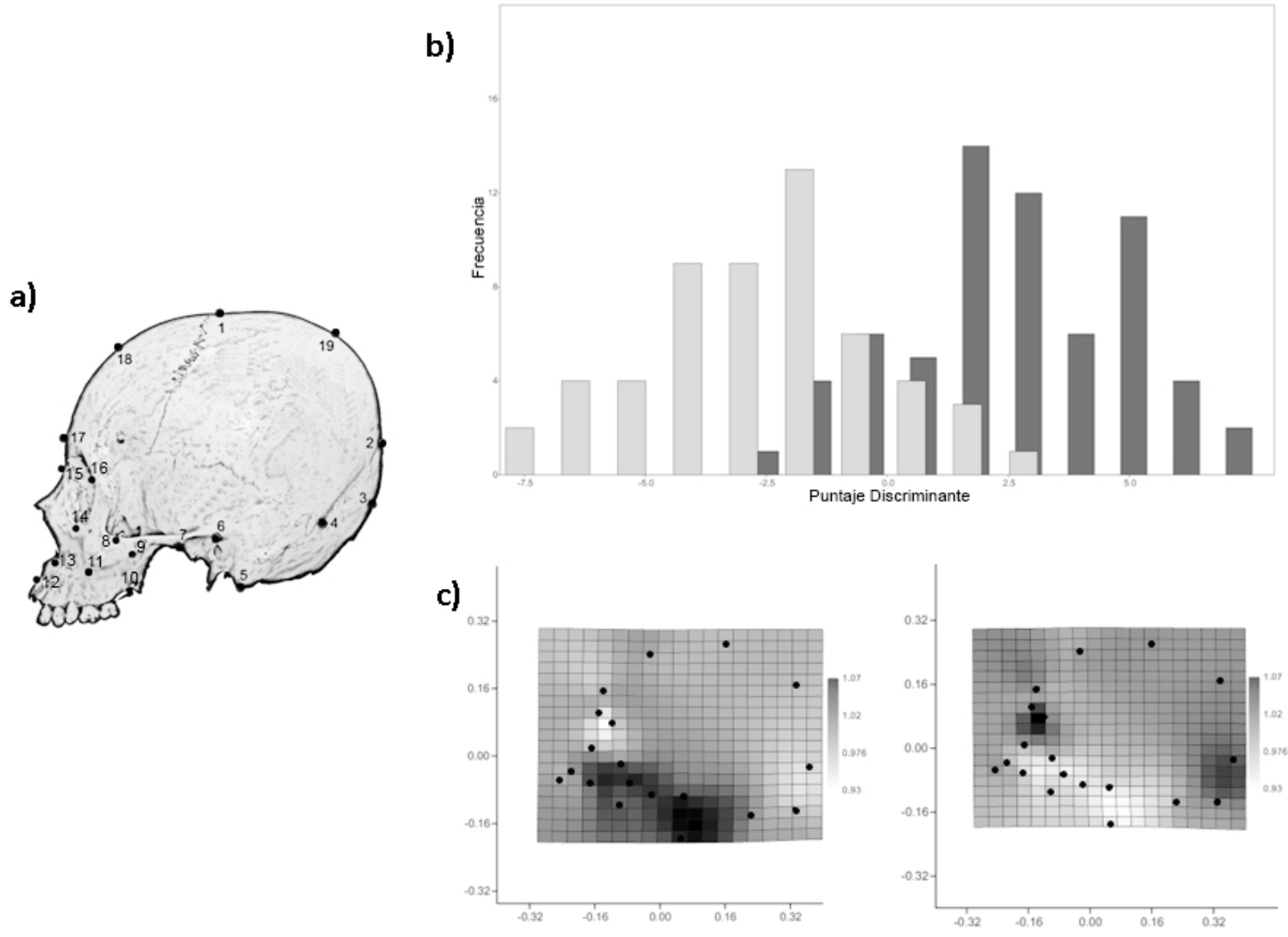

Fig. 4. Ejemplo de aplicación de la MG para el análisis del dimorfismo sexual: a) Vista lateral de un cráneo humano arqueológico mostrando los 19 hitos utilizados; b) Gráfico de clasificación de la puntajes para los 120 individuos analizados tras la estimación de una función discriminante, (gris oscuro: individuos femeninos; gris claro: individuos masculinos), se observa un esperable sobrelapamiento para una especie con un moderado nivel de dimorfismo sexual, aunque se encontraron diferencias significativas entre los grupos (Hotelling's T2: 173.4452 ; p-valor: $<0,001 ; 86,2 \%$ y $85,5 \%$ de individuos correctamente clasificados para individuos femeninos y masculinos respectivamente); c) Función de placa delgada (TPS) entre el consenso poblacional y los consensos masculino (izquierda) y femenino (derecha). Los puntos negros representan los hitos, las grillas representan las deformaciones entre los especímenes, mientras que la escala de grises representa a los factores de expansión (colores más oscuros son indicativos de una mayor expansión local). Se observa que gran parte de las diferencias entre sexos se concentran en las área mastoidal y rostral, con una mayor expansión de estas zonas en los especímenes masculinos. 
La evolución del dimorfismo sexual ha sido ampliamente documentada; sin embargo, la mayoría de los estudios se han referido a la diferencia del tamaño entre sexos opuestos, mientras que la variación de la forma ha sido mucho menos investigada (Lande, 1980; Lande \& Arnold, 1985; Hedrick \& Temeles, 1989; Fairbairn et al., 2007). No obstante, la mayoría de los estudios del dimorfismo sexual de la forma, han tratado a este ultimo como un carácter diagnostico para identificación sexual o en análisis ontogenéticos (Ohiggins et al., 1990; Pretorius, 2005; Valenzuela et al., 2004). Mas aun, las investigaciones que han considerado la evolución del dimorfismo sexual, han cubierto sólo algunos aspectos en un limitado número de taxa como: la evolución del cráneo en primates (Leigh, 2006; Schaefer et al., 2004), las proporciones y dimensiones en cuerpos de lagartos (Butler \& Losos, 2002; Butler et al., 2007), tritones (Malmgren \& Thollesson, 1999) o en moscas (Bonduriansky, 2006), y la variación de la forma en las cabezas en insectos (Atchley, 1971), y la variación del dimorfismo sexual en las alas de Drosophila (Gidaszewski et al., 2009) o de la forma corporal en escarabajos (Benítez De La Fuente et al.; Benítez et al., 2013).

Se ha sugerido que el nivel de dimorfismo sexual esqueletal en cráneo humanos es relativamente moderado o leve en comparación a otros primates (Frayer \& Wolpoff, 1985; O'Higgins et al.; Rosas \& Bastir, 2002). Variadas líneas de evidencia sustentan que existe una estrecha relación entre las estrategias socio-reproductivas y el nivel de dimorfismo sexual (Kappeler \& van Schaik, 2002). En la presente revisión decidimos analizar la clásica problemática acerca del nivel de dimorfismo sexual en la forma de cráneos humanos, utilizando para esto las herramientas de la MG 2D (Fig. 4). Una muestra de 120 cráneos humanos (femeninos: 65; masculinos: 55) fue fotografiada de manera estandarizada en vista lateral, y las diferencias debidas a escala, traslación y rotación fueron removidas mediante un análisis de Procusto. Tras esto, se realizó un análisis discriminante para distinguir entre individuos masculinos y femeninos. Nuestros resultados muestran que existen diferencias significativas entre ambos sexos (Hotelling's T2: 173.4452; pvalor: $<0,001)$, no obstante un esperable sobrelapamiento para una especie con moderado dimorfismo sexual a nivel morfológico esqueletal $(86,2 \%$ y $85,5 \%$ de individuos correctamente clasificados para individuos femeninos y masculinos respectivamente). La función de TPS mostró que gran parte de las diferencias entre sexos se concentran en las área mastoídea y rostral, con una mayor expansión de estas zonas en los especímenes masculinos. En concordancia con lo recién señalado, tam- bién hubo diferencias significativas en el tamaño del centroide entre ambos sexos (t-test: -2.6479; p-valor: $0,009)$, siendo los individuos femeninos más pequeños.

\section{CONCLUSIONES}

La incorporación de nuevas técnicas que determinan y sectorizan la variación de la forma tanto para animales o plantas esta siendo actualmente un tema muy debatido (Lawing \& Polly, 2010, Adams et al.). La MG se presenta como una posible solución, debido a su capacidad de unificar e incorporar diferentes metodologías las cuales se unifican para cuantificar y visualizar la forma de múltiples maneras. Diversos trabajos han establecido relaciones entre la asimetría fluctuante de la forma y las variaciones en la diversidad genética. No obstante estas asociaciones son relativamente leves, probablemente debido a la ausencia de un método estandarizado a seguir. Una opción que los científicos están siguiendo, es combinar estas técnicas avanzadas morfométricas, unificando metodologías junto a estudios moleculares y genéticos, para así poder obtener resultados con evidencia total dentro de los análisis, No obstante estudios que mezclan patrones de movimiento y biomecánica también son parte importante del crecimiento de este nicho de investigación (Adams et al.).

Esta revisión pretendió entregar una perspectiva amplia del uso de la MG para algunos de los diferentes ámbitos de la biología evolutiva, propugnando la necesidad de aumentar el esfuerzo investigativo en esta disciplina, junto con llamar la atención acerca de la utilidad de la MG como una herramienta efectiva, precisa, amigable y barata para cuantificar y estudiar la variación morfológica en una escala evolutiva.

\section{AGRADECIMIENTOS}

Agradecemos a Philippe Menecier (Musée de l'Homme, Paris) y a Manuel Arturo Torres (Museo G. Le Paige, San Pedro de Atacama) por el acceso a los especímenes arqueológicos bajo su cuidado. Gracias también a Germán Manríquez por el apoyo a esta investigación y a Stephan Püschel y Katherine Avendaño por la edición de algunas de las figuras aquí presentadas. Este trabajo fue financiado parcialmente por el Programa de Investigación Asociativa Anillos en Ciencia y Tecnología ACT No 096 y por Becas Chile, Conicyt-PCHA/2012/73130010 (TP). 
BENÍTEZ, H. A. \& PÜSCHEL, T. A. Modelling shape variance: geometric morphometric applications in evolutionary biology. Int. J. Morphol., 32(3):998-1008, 2014.

SUMMARY: The comparison of anatomical traits between organisms has been a central topic in comparative biology. Historically, taxanomic classification and biological diversity understanding have been based on morphological descriptions. Derived from a mathematical quantitative revolution, morphological studies have experienced an important renewal due to the development of shape analysis rooted in statistical multivariate methods and novel visualisation techniques. The aim of the present review is to provide an updated perspective regarding the progress in geometric morphometrics (GMM) applied to evolutionary biology, as well as introducing to cutting-edge subjects (e.g. developmental stability, modularity, morphological integration, among other themes). Thus, it is expected to provide a broad point of view with respect to the appliaction of geometric morphometrics in evolutionary biology, highlighting its usefulness as an effective, accurate, user-friendly and inexpensive method to quantify and study shape variation.

KEY WORDS: Geometric morphometrics; Evolutionary biology; Integration; Modularity; Fluctuating asymmetry; Sexual dimorphism.

\section{REFERENCIAS BIBLIOGRAFICAS}

Ackermann, R. R. Ontogenetic integration in the hominoid face. $J$. Hum. Evol., 48(2):175-97, 2005.

Adams, D. C. \& Funk, D. J. Morphometric inferences on sibling species and sexual dimorphism in neochlamisus bebbianae leaf beetles: multivariate applications of the thin-plate spline. Syst. Biol., 46(1):180-94, 1997.

Adams, D. C. \& Rohlf, F. J. Ecological character displacement in Plethodon: biomechanical differences found from a geometric morphometric study. Proc. Natl. Acad. Sci. USA, 97(8):410611, 2000.

Adams, D. C.; Rohlf, F. J. \& Slice, D. E. Geometric morphometrics: ten years of progress following the 'revolution'. Ital. J. Zool., 71:5-16, 2004.

Adams, D. C.; Rohlf, F. J. \& Slice, D. E. A field comes of age: geometric morphometrics in the 21st century. Hystrix, 24(1):7$14,2013$.

Alibert, P.; Moureau, B.; Dommergues, J. L. \& David, B. Differentiation at a microgeographical scale within two species of ground beetle, Carabus auronitens and C. nemoralis (Coleoptera, Carabidae): a geometrical morphometric approach. Zool. Scr., 30(4):299-311, 2001.

Atchley, W. R. Components of sexual dimorphism in chironomus larvae (Diptera: Chironomidae). Am. Nat., 105(945):455-66, 1971.

Atchley, W. R.; Gaskins, C. T. \& Anderson, D. Statistical Properties of Ratios. I. Empirical Results. Syst. Biol., 25(2):137-48, 1976.

Auffray, J-C.; Debat, V. \& Alibert, P. Shape asymmetry and developmental stability. In: Chaplain, M. A. J.; Singh, G. D. \& McLachlan, J. C. (Eds.). On growth and form: spatio-temporal pattern formation in biology. Chichester, John Wiley \& Sons Ltd., 1999. pp.309-24.
Bastir, M. \& Rosas, A. Hierarchical nature of morphological integration and modularity in the human posterior face. Am. J. Phys. Anthropol., 128(1):26-34, 2005.

Benítez, H. A. Assessment of patterns of fluctuating asymmetry and sexual dimorphism in carabid body shape. Neotrop. Entomol., 42(2):164-9, 2013.

Benítez, H. A.; Avaria-Llautureo, J.; Canales-Aguirre, C. B.; Jerez, V.; Parra, L. E. \& Hernández, C. E. Evolution of sexual size dimorphism and its relationship with sex ratio in carabid beetles of Genus Ceroglossus Solier. Curr. Zool., 59(6):769-77, 2013.

Benítez. H. A.; Parra, L. E.; Sepulveda, E. \& Sanzana, M. J. Geometric Perspectives of Sexual Dimorphism in the Wing Shape of Lepidoptera: the Case of Synneuria sp. (Lepidoptera: Geometridae). J. Entomol. Res. Soc., 13(1):53-60, 2011.

Benítez De La Fuente, H.; Vidal, M.; Briones, R. \& Jerez, V. Sexual Dimorphism and Morphological Variation in Populations of Ceroglossus Chilenisch (Eschscholtz, 1829)(Coleoptera: Carabidae). J. Entomol. Res. Soc., 12(2):87-95, 2010.

Bertin, A.; David, B.; Cezilly, F. \& Alibert, P. Quantification of sexual dimorphism in Asellus aquaticus (Crustacea: Isopoda) using outline approaches. Biol. J. Linn. Soc., 77:523-33, 2002.

Bonduriansky, R. Convergent evolution of sexual shape dimorphism in Diptera. J. Morphol., 267(5):602-11, 2006.

Bookstein, F. L. Morphometric Tool for Landmark Data. Geometry and Biology. Cambridge, Cambridge University Press, 1991.

Bookstein, F. L. A hundred years of morphometrics. Acta Zool. Hung., 44:7-59, 1998.

Bravi, R. \& Benítez, H. A. Left-right asymmetries and shape analysis on Ceroglossus chilensis (Coleoptera: Carabidae). Acta Oecol., 52:57-62, 2013. 
Brown, L. R.; Moyle, P. B.; Bennett, W. A. \& Quelvog, B. D. Implications of morphological variation among populations of California roach Lavinia symmetricus (Cyprinidae) for conservation policy. Biol. Conserv., 62(1):1-10, 1992.

Butler, M. A. \& Losos, J. B. Multivariate sexual dimorphism, sexual selection, and adaptation in Greater Antillean Anolis lizards. Ecol. Monogr., 72(4):541-59, 2002.

Butler, M. A.; Sawyer, S. A. \& Losos, J. B. Sexual dimorphism and adaptive radiation in Anolis lizards Nature, 447(7141):202-5, 2007.

Cantín, M.; Salgado, G.; Inzunza, O.; Inostroza, V.; Errázuriz, M. J.; Pavez, C. \& Fuentes, R. Direct morphometric evaluation of condylar asymmetry on human mandibles. Int. J. Morphol., 31(4):1401-6, 2013.

Cheverud, J. M.; Dow, M. M. \& Leutenegger, W. The quantitative assessment of phylogenetic constraints in comparative analyses: sexual dimorphism in body weight among primates. Evolution, 39(6):1335-51, 1985.

Cheverud, J. M.; Rutledge, J. J. \& Atchley, W. R. Quantitative genetics of development: genetic correlations among agespecific trait values and the evolution of ontogeny. Evolution, 37(5):895-905, 1983.

Clarke, G. M. Developmental stability and fitness: the evidence is not quite so clear. Am. Nat., 152(5):762-6, 1998.

Darroch, J. N. \& Mosimann, J. E. Canonical and principal components of shape. Biom., 72(2):241-52, 1985.

Debat, V.; Debelle, A. \& Dworkin, I. Plasticity, canalization, and developmental stability of the Drosophila wing: joint effects of mutations and developmental temperature. Evolution, 63(11):2864-76, 2009.

Dryden, I. L. \& Mardia, K. V. Statistical Shape Analysis. Chichester, John Wiley and Sons Ltd., 1998.

Fairbairn, D. J. \& Preziosi, R. F. Sexual selection and the evolution of sexual size dimorphism in the water strider, Aquarius remigis. Evolution,50(4):1549-59, 1996.

Fairbairn, D. J.; Blanckenhorn, W. U. \& Székely, T. (Eds.). Sex, size and gender roles: Evolutionary studies of sexual size dimorphism. Oxford, Oxford University Press, 2007.

Fondon, J. W. 3rd. \& Garner, H. R. Detection of lengthdependent effects of tandem repeat alleles by 3-D geometric decomposition of craniofacial variation. Dev. Genes Evol., 217(1):79-85, 2007.

Frayer, D. W. \& Wolpoff, M. H. Sexual Dimorphism. Annu. Rev. Anthropol., 14:429-73, 1985.
Galdames, I. S. \& Zavando, D. Age effect in the morphological traits performance for sex determination in human skulls and mandibles. Int. J. Morphol., 30(1):296-301, 2012.

Gannon, W. L. \& Rácz, G. R. Character displacement and ecomorphological analysis of two long-eared Myotis ( $M$. auriculus and M. evotis). J. Mammal., 87(1):171-9, 2006.

Gidaszewski, N. A.; Baylac, M. \& Klingenberg, C. P. Evolution of sexual dimorphism of wing shape in the Drosophila melanogaster subgroup. B.M.C. Evol. Biol., 9:110, 2009.

Goodall, C. Procrustes methods in the statistical-analysis of shape. J. R. Stat. Soc. B., 53(2):285-339, 1991.

Goswami, A. \& Polly, P. D. Methods for studying morphological integration and modularity. In: Alroy, J. \& Hunt, G. (Eds.). Quantitative methods in paleobiology. Paleontol. Soc. Pap., 16:213-43, 2010.

Hallgrimsson, B. \& Hall, B. K. Variation: A Central Concept in Biology. San Diego, Elsevier Academic Press, 2005.

Hedrick, A. V. \& Temeles, E. J. The evolution of sexual dimorphism in animals: Hypotheses and tests. Trends Ecol. Evol., 4(5):136$8,1989$.

Kappeler, P. M. \& van Schaik, C. P. Evolution of primate social systems. Int. J. Primatol., 23(4):707-40, 2002.

Klingenberg, C. P. Cranial integration and modularity: insights into evolution and development from morphometric data. Hystrix, 24(1):43-58, 2013.

Klingenberg, C. P. Morphological integration and developmental modularity. Annu. Rev. Ecol. Evol. Sys., 39:115-32, 2008.

Klingenberg, C. P. Morphometric integration and modularity in configurations of landmarks: tools for evaluating a priori hypotheses. Evol. Dev., 11(4):405-21, 2009.

Klingenberg, C. P. Integration, modules and development: molecules to morphology to evolution. In: Pigliucci, M. \& Preston, K. (Eds.). Phenotypic Integration: Studying the Ecology and Evolution of Complex Phenotypes. New York, Oxford University Press, 2004. pp.213-30.

Klingenberg, C. P. Developmental instability as a research tool: using patterns of fluctuating asymmetry to infer the developmental origins of morphological integration. In: Polak, M. (Ed.). Developmental Instability: Causes and Consequences, New York, Oxford University Press, 2003. pp.427-42.

Klingenberg, C. P, \& Marugán-Lobón, J. Evolutionary covariation in geometric morphometric data: analyzing integration, modularity, and allometry in a phylogenetic context. Syst. Biol., 62(4):591-610, 2013. 
Klingenberg, C. P. \& Zaklan, S. D. Morphological integration between development compartments in the Drosophila wing. Evolution, 54(4):1273-85, 2000.

Klingenberg, C. P. \& Leamy, L. J. Quantitative genetics of geometric shape in the mouse mandible. Evolution, 55(11):2342-52, 2001.

Klingenberg, C. P. \& McIntyre, G. S. Geometric morphometrics of developmental instability: analyzing patterns of fluctuating asymmetry with Procrustes methods. Evolution, 52:1363-75, 1998.

Klingenberg, C. P.; Mebus, K. \& Auffray, J C. Developmental integration in a complex morphological structure: how distinct are the modules in the mouse mandible? Evol. Dev., 5(5):522-31, 2003.

Lande, R. Genetic-variation and phenotypic evolution during allopatric speciation. Am. Nat., 116(4):463-79, 1980.

Lande, R., \& Arnold, S. J. Evolution of mating preference and sexual dimorphism. J. Theor. Biol., 117(4):651-64, 1985.

Lawing, A. M. \& Polly, P. D. Geometric morphometrics: recent applications to the study of evolution and development. $J$. Zool., 280(1):1-7, 2010.

Leigh, S. R. Cranial ontogeny of Papio baboons (Papio hamadryas). Am. J. Phys. Anthropol., 130(1):71-84, 2006.

Lele, S. R. \& Richtsmeier, J. T. An Invariant Approach to Statistical Analysis of Shapes. New York, Chapman and Hall/ CRC, 2001.

López, B.; Toro, V.; Schilling, A. \& Galdames, I. S. Nasal Profile Assessment Using Geometric Morphometrics in a Sample of Chilean Population: Clinical and Forensic Implications. Int. J. Morphol., 30(1):302-8, 2012.

MacLeod, N. \& Forey, P. (Eds.). Morphology, shape, and phylogeny. London, Taylor \& Francis, 2002.

MacLeod, N. Principal Components Analysis (Eigenanalysis \& Regression 5). Palaeontol. Assoc. Newsl., 59:42-54, 2005.

Malmgren, J. C. \& Thollesson, M. Sexual size and shape dimorphism in two species of newts, Triturus cristatus and T. vulgaris (Caudata: Salamandridae). J. Zool., 249(2):12736, 1999.

Marcus, L. F. Traditional Morphometrics. In: Rohlf, F. J. \& Bookstein, F. L. (Eds.) Proceedings of the Michigan Morphometrics Workshop. Ann Arbor, University of Michigan Museum of Zoology Special Publication 2, 1990.

McKinney, M. L. \& McNamara, K. J. Heterochrony: The Evolution of Ontogeny. New York, Springer, 1991.
Mitteroecker, P. \& Bookstein, F. The conceptual and statistical relationship between modularity and morphological integration. Syst. Biol., 56(5):818-36, 2007.

Mitteroecker, P. \& Bookstein, F. The evolutionary role of modularity and integration in the hominoid cranium. Evolution, 62(4):943-58, 2008.

Monteiro, L. R. \& Nogueira, M. R. Adaptive radiations, ecological specialization, and the evolutionary integration of complex morphological structures. Evolution, 64(3):724-44, 2010.

Monteiro, L. R. \& Nogueira, M. R. Evolutionary patterns and processes in the radiation of phyllostomid bats. B. M. C. Evol. Biol., 11:137, 2011.

Nijhout, H. F. \& Davidowitz, G. Developmental perspectives on phenotypic instability, canalization, and fluctuating asymmetry. In: Polak, M. Developmental Instability: Causes and Consequences. Oxford, Oxford University Press, 2003 .

Nogueira, M. R.; Peracchi, A. L. \& Monteiro, L. R. Morphological correlates of bite force and diet in the skull and mandible of phyllostomid bats. Funct. Ecol., 23(4):715-23, 2009.

O'Higgins, P. The study of morphological variation in the hominid fossil record: biology, landmarks and geometry. J. Anat., 197 (Pt. 1):103-20, 2000

O’Higgins, P.; Moore, W. J.; Johnson, D. R.; McAndrew, T. J. \& Flinn, R. M. Patterns of cranial sexual dimorphism in certain groups of extant hominoids. J. Zool., 222(3):399-420, 1990.

Olson, E. C. \& Miller, R. L. Morphological integration. Chicago, University of Chicago Press, 1958.

Palmer, A. R. \& Strobeck, C. Fluctuating asymmetry: measurement, analysis, patterns. Ann. Rev. Ecol. Syst., 17:391-421, 1986.

Parés-Casanova, P. M. \& Martínez, S. Geometric Morphometrics for the Study of Hemicoxae Sexual Dimorphism in a Local Domestic Equine Breed. Int. J. Morphol., 31(2):623-8, 2013.

Pither, J. \& Taylor, P. D. Directional and fluctuating asymmetry in the black-winged damselfly Calopteryx maculata (Beauvois)(Odonata: Calopterygidae). Can. J. Zool., 78(10):1740-8, 2000

Pigliucci, M. \& Preston, K. Phenotypic Integration: Studying the Ecology and Evolution of Complex Phenotypes. New York, Oxford University Press, 2004.

Pretorius, E. Using geometric morphometrics to investigate wing dimorphism in males and females of Hymenoptera - a case study based on the genus Tachysphex Kohl (Hymenoptera: Sphecidae: Larrinae). Aust. J. Entomol., 44(2):113-21, 2005. 
Polak, M. Developmental Instability: Causes and. Consequences. New York, Oxford University Press, 2003. p.459.

Rieppel, O. 'Type' in morphology and phylogeny. J. Morphol., 267(5):528-35, 2006.

Rios-Rodas, L.; Rodríguez-Romero, F. J.; Velázquez-Rodríguez, A. S. \& Hernández-Franyutti, A. A. Geometric Morphometric of the Heart in Hyla plicata Through an Altitudinal Gradient in the Mexican Neovolcanic Axe. Int. J. Morphol., 31(3):905$10,2013$.

Rohlf, F. J.; Loy, A. \& Corti, M. Morphometric analysis of old world talpidae (Mammalia, Insectivora) using partial-warp scores. Syst. Biol., 45(3):344-62, 1996.

Rohlf, F. J. \& Marcus, L. F. A revolution in morphometrics. Trends Ecol. Evol., 8(4):129-32, 1993.

Rohlf, F. J. \& Slice, D. Extensions of the procrustes method for the optimal superimposition of landmarks. Syst. Zool., 39(1):4059, 1990.

Rosas, A. \& Bastir, M. Thin-plate spline analysis of allometry and sexual dimorphism in the human craniofacial complex. Am. J. Phys. Anthropol., 117(3):236-45, 2002.

Schaefer, K.; Mitteroecker, P.; Gunz, P.; Bernhard, M. \& Bookstein, F. L. Craniofacial sexual dimorphism patterns and allometry among extant hominids. Ann. Anat., 186(5-6):471-8, 2004.

Shea, B. T. Bivariate and multivariate growth allometry: statistical and biological considerations. J. Zool., 206(3):367-90, 1985.

Slice, D. E. Modern Morphometrics in Physical Anthropology. New York, Kluwer Academic Publishers, 2005.

Slice, D. E. Geometric morphometrics. Annu. Rev. Anthropol., 36:261-81, 2007.

Small, C. G. The statistical theory of shape. New York, SpringerVerlag, 1996.

Thomas, C. D.; Hill, J. K. \& Lewis, O. T. Evolutionary consequences of habitat fragmentation in a localized butterfly. J. Anim. Ecol., 67(3):485-97, 1998.

Thompson, D. W. On growth and form. 2nd ed. New York, Cambridge University Press, 1942.

Toro Ibacache, M. V.; Manriquez Soto, G. \& Suazo Galdames, I. Geometric morphometry and the biologic shapes study: from the descriptive morphology to the quantitative morphology. Int. J. Morphol., 28(4):977-90, 2010.

Tornese, E. B. \& Nabar, M. J. M. Morphometry of the corpus callosum and mammillary bodies in alcoholism using magnetic resonance. Int. J. Morphol., 31(4):1233-42, 2013.
Valenzuela, N.; Adams, D. C.; Bowden, R. M. \& Gauger, A. C. Geometric morphometric sex estimation for hatchling turtles: a powerful alternative for detecting subtle sexual shape dimorphism. Copeia, 2014(4):735-42, 2004.

Van Valen, L. A study of fluctuating asymmetry. Evolution, 16(2):125-42, 1962.

Waddington, C. H. Canalization of development and the inheritance of acquired characters. Nature, 150:563-5, 1942.

Wagner, G. P. On the eigenvalue distribution of genetic and phenotypic dispersion matrices: Evidence for a nonrandom organization of quantitative character variation. J. Math. Biol., 21(1):77-95, 1984.

Wagner, G. P. A comparative study of morphological integration in Apis mellifera (Insecta, Hymenoptera). J. Zool. Syst. Evol. Res., 28(1):48-61, 1990.

Willmore, K. E.; Leamy, L. \& Hallgrímsson, B. Effects of developmental and functional interactions on mouse cranial variability through late ontogeny. Evol. Dev., 8(6):550-67, 2006.

White, T. A. \& Searle, J. B. Mandible asymmetry and genetic diversity in island populations of the common shrew, Sorex araneus. J. Evol. Biol., 21(2):636-41, 2008.

Young, N. M. Function, ontogeny and canalization of shape variance in the primate scapula. J. Anat., 209(5):623-36, 2006.

Zelditch, M. L.; Swiderski. D. L.; Sheets, H. D. \& Fink, W. L. Geometric Morphometrics for Biologists. A Primer. London, Elsevier, 2004.

Zelditch, M. L.; Wood, A. R.; Bonett, R. M. \& Swiderski, D. L. Modularity of the rodent mandible: integrating bones, muscles, and teeth. Evol. Dev., 10(6):756-68, 2008.

Zelditch, M. L.; Swiderski, D. L.; Sheets, D. \& Fink, W. L. Geometric Morphometrics for Biologists. A Primer. London, Elsevier, 2012.

\section{Correspondence to: \\ Dr. Hugo Benitez \\ Faculty of Life Sciences \\ University of Manchester \\ Michael Smith Building \\ Oxford Road, Manchester M13 9PT \\ UNITED KINGDOM}

Email: hugo.benitez@postgrad.manchester.ac.uk

Recibido : 22-09-2013

Aceptado: 17-12-2014 